\title{
Energy-Efficient Heterogeneous Optimization Routing Protocol for Wireless Sensor Network
}

\author{
Srilakshmi Uppalapati \\ Department of CSE, VFSTR Deemed to university, Vadlamudi, Guntur 522213, A.P, India
}

Corresponding Author Email: usl_cse@vignan.ac.in

https://doi.org/10.18280/i2m.190510

Received: 20 July 2020

Accepted: 5 October 2020

\section{Keywords:}

wireless sensor networks, distance, scheduling, routing protocol, E-BEENISH, EE-TDMA-PSOUFC

\begin{abstract}
A wide range of applications include in Wireless Sensor Networks (WSNs), and it is being used extensively in data collection specifically to process the mission-critical tasks. The implementation of routing protocols of energy-efficient (EE) is one of the significant challenging jobs of Sensor Networks (MC-SSN) and Mission Critical Sensors. In hierarchical routing protocols, higher EE can reach when compared to the flat routing protocols. The network's scheduling process doesn't support enhanced balanced Energyefficient network-integrated super-heterogeneous (E-BEENISH), which discusses earlier. An Energy. Energy efficient Time scheduling based particle swarm optimization unequal fault tolerance clustering protocol (EE-TDMA-PSO-UFC) is proposed in this paper. Based on the distance parameter, an efficient cluster head $(\mathrm{CH})$ is selected in this protocol. Owing to the unexpected failure of $\mathrm{MCH}$ (Master Cluster Head), an additional "CH" is chosen that is termed as Surrogate cluster head $(\mathrm{SCH})$ for the restoration of network's connectivity in the protocol of PSO-UFC. Based on TDMA (Time Division Multiple Access) protocols, the consumption of Energy. Energy is reduced with the allocation of timeslots during transmission of data. Using the technique of EE-TDMA-PSOUFC, the network's lifespan improves than CEEC and E-BEENISH protocols according to the assessment of simulation results.
\end{abstract}

\section{INTRODUCTION}

Sensor Networks and Mission Critical Sensors have provided numerous connections of sensors and devices in various applications [1]. However, different applications exist for WSNs and are being exploited to collect data and perform mission-critical tasks efficiently. Here, the mission-critical tasks are involved an enormous number of small autonomous devices, which are known as nodes that are dealing with information sensing, data processing, and creating a communication link between nodes [2, 3]. In general, the energy conservation in WSNs is more due to the massive number of nodes of sensors and the requirement of processing of data, monitoring, and other relevant activities. The nodes are getting charged by batteries in WSNs. A vital parameter is considered as energy consumption in WSNs. For reducing energy conservation and improve the network's lifetime, a variety of techniques, algorithms, and protocols have been implemented. For detection or measuring the environmental conditions, the deployment of WSNs is done. The environmental conditions are included traffic control, home automation, environment or habitat monitoring, etc. [4-7]. As WSNs have benefits in different fields, it is a great interest to full fill the activities rapidly in daily life. Because the sensor nodes have restricted availability of energy resources [8-12], it's not required to operate a network in a normal state as the sensor nodes are involved with the sporadic detection of events

In WSNs $[13,14]$, two kinds of environments are existed, such as heterogeneous and homogeneous. Low-energy adaptive clustering methods, various heterogeneous routing protocols, and energy management are available in recent times [15-17]. According to each node's residual energy, the probability of " $\mathrm{CH}$ " is chosen in the stable election protocol (SEP). Before the first node's death, the time interval is extended by the residual energy [18]. A clustering scheme of "EE" has been proposed and processed by Qing et al. for one wireless sensor network of the heterogeneous type, which is termed as distributed energy-efficient clustering (DEEC) [19]. The probability of selection of "CH" differs between SEP and DEEC schemes. The "CH" is chosen based on the ratio's probability function to the residual energy to the network's average power. By relying on the node's residual energy and initial energy, the duration to become the "CH." For three different types of nodes, enhanced DEEC (E-DEEC) is proposed by Saini et al. that helps to increase the lifetime and network's stability [20], which involves the addition of another node named as a supernode by increasing the heterogeneity and following the philosophy of DEEC. A routing protocol of a centralized energy-efficient clustering (CEEC) was demonstrated, as mentioned in the study [21]. Three equal areas are categorized as the network by "CEEC," where the distribution of nodes with the same energy in the same place is done. The protocol of the balanced energy-efficient network integration super heterogeneous (BEENISH) [22] was developed, which is led by the heterogeneous WSNs' energy levels with a wide range and the cluster after assessing the communication's energy conservation. BEENISH protocol assumes choosing "CH" with the use of involved four-node levels and node's residual energy in WSNs.

The key points of the literature review as follows: 
$>$ By considering the distance between selected cluster members and cluster, an algorithm is formulated to restrict the threshold setting of EE-TDMA-PSOUFC protocol. The process is not complicated.

$>$ Based on the factor of distance, the selection of different types of "CHs" like "SCH" and " $\mathrm{MCH}$ " is developed for supporting the network's lifecycle to curb the effect of ping-pong.

\section{LITERATURE SURVEY}

While selecting the CHs, the distance effect didn't employ in the discussed protocols referred to by Verma et al. [23] that is a significant factor in achieving a network's stability. The factors like energy and distance, the selection process of $\mathrm{CHs}$ is getting affected using a neural network. The optimization of the network's number of clusters and sensor nodes' energy conservation is done in the neural network through the training of neurons.

The author proposed a new multiple chain scheme [24], and it is called MAMC (Multiple Aggregator Multiple Chain), in which ten regions are categorized in the network based on the chain in every region operating as PEGASIS. The aggregator node gathers the information from nodes, and it is transmitted to the sink node. The equal distribution of energy load over the entire network is possible when it is divided into multiple chains. Hence, the sensor network's performance is improved. The network's heterogeneity has various benefits, which have been shown in this paper.

A secure protocol known as E-STAR is demonstrated by the Mahmoud et al. [25] to achieve reliable and stable routes in HMWNs. Based on an energy-aware and trust-based routing protocol, the trust and payment systems are integrated by ESTAR. For energizing the nodes, the credits or micropayment are utilized by the payment system. Here, the nodes are used to send the packets and reward the relaying packets. To manage the credit accounts of nodes, an offline trusted party (TP) is necessary as it may not be included in communication sessions. The relaying packets' proofs are contained in the nodes, and they are termed as receipts which used to submit to TP. The selfish nodes can be simulated by the payment system for earning the credits to relay on others' packets. With the rewarding of nodes, fairness can be enforced that relay more packets available at the network centre. For ensuring the route stability, the payment system is not enough. The rational nodes can be simulated by the payment system to earn the credits, not split the routes. Due to some other reasons like a node's failure, low resources, and malicious attacks, the routes may be broken.

For the field of multi-level energy heterogeneous sensors, a novel hierarchical clustering routing protocol (MLEEC) is described by Al Masri et al. [26]. The improved throughput of a network is the main objective of the proposed protocol. It means that the total data packets are received at BS and a network lifetime, i.e., the calculation of a time until the death of the last node and the decreasing and balancing the energy dissipation between the network's nodes.

Srilakshmi [27] was proposed and developed an IDS that facilitates the foolproof security to MANET and improves routing performance, delay, and packet delivery ratio. Without inculcating a fixed infrastructure, nodes are collected and configured automatically by a Mobile Ad Hoc Network (MANET). In case of emergencies where non-availability of fixed infrastructure networks, MANET can be utilized. Vital importance is given to the securing of MANET communication. Both roles, like receiver and transmitter, are played by the nodes in MANET. In protecting MANET communication, intrusion detection is playing a significant role, and various IDSs exist.

SriLakshmi [28] introduced a qualified learns of Secure Intrusion-Detection Systems to decide the noxious hubs and assaults on MANETs. To deal with the safety systems, aversion instruments of MANETs are only not enough due to some exceptional qualities of MANETs. The information correspondence between various gatherings and their versatility is allowed as one of the principles of favourable circumstances. This message is a scarcity to the transmitter's scope.

Srilakshmi [29] was attempted to resolve this issue by designing a new intrusion detection system named Random Walk Detector (RWD) based approach specially designed for MANETs. The algorithm is formulated based on the data fusion technique. ECC mechanism is added to achieve the data integrity feature. Simulation results are provided, showing that in the presence of malevolent-node attacks, the RWDB outperforms the EAACK, CBDS, and ECDSA in terms of packet delivery ratio, throughput, and routing overhead.

Srilakshmi and Rao [30] presents the protocol that incorporates link failure prediction at the network layer and Power Control Protocol at the MAC layer to improve network performance. Simulation results show performance enhancement regarding total power transmission, energy regulation, consumption per node, and throughput of our proposed cross-layer routing protocol compared to AODV.

Srilakshmi and Rao [30] presents the Mobile ad hoc network design is for making the availability of Internet facility at the locations and every second despite the consequences of geographical location. The resourcecontrolled situation of MANET would build its communication procedures very tricky. Additionally, the network nodes are prepared along with batteries that are inhibited $\&$ it is more difficult for replacing or recharging the batteries at the time of the mission. The accumulation of node in MANET is finished whatever be the circumstances; for the node for communicating with another node, there should be a safe \& dependent technique.

Srilakshmi et al. [31] that the mobility \& nodes congestion would lead to recurrent failures of link and the shortcomings of packet that will affect the QoS protocol performance. BeeAd-Hoc system is being commenced as the most appropriate technique and a proper load balance for increasing the MANET structure's efficient energy. We would be utilized a useful suggested method, Load Balanced and Energy Efficient Bee-Ad-Hoc Multipath Routing with Routing Transmission in MANET, to overcome previous methods and Restrictions in mobile ad hoc networks within this work. This method would be maximizing an end-and-and connectivity in the network and also would reduce the faults at link or / and level of node.

\section{PROPOSED SYSTEM}

EE-TDMA-PSO-UFC protocol is categorized into rounds involving two phases: a steady-state phase and a cluster setup phase. Clusters are generated in a setup phase, and the data is transmitted to the cluster head in the steady-state phase. $\mathrm{N}$ frames are divided into the EE-TDMA-PSO-UFC's steady- 
state phase. Each frame's duration is not determined because each source node doesn't have the data for sending it always. A period of data transmission and a schedule period are contained in each frame. For collision-free intracluster communication, a TDMA schedule is setup by the cluster head during each program period. A similar time slot is assigned to cluster members in each round's first schedule. Different types of time slots are allocated to the source nodes in the other schedule period. The time slots are categorized by every data transmission period, and the time slots are equalized to the source nodes' number in a cluster. Each source node sends the data, and the next frame's traffic load is expected to the cluster head over the assigned time slot of transmission. All the other times, the radio is kept to the off condition. In the period of data transmission, all nodes of non-source have included the state of radios off. The information may send by the source node that involves leaving a message to the cluster head. Based on the sensor nodes' number and traffic load of the sensor node, the TDMA frame's length can be changed by the cluster head dynamically. A scheduled period is initiated by the next frame when a structure accomplishes, and a similar procedure is repeated. From all types of source nodes, the cluster head gathers the data, and the compressed and aggregated information is forwarded to the BS. The system is initiated in the next round after a predefined period, and the whole procedure repeats. In Figure 2, the proposed system's flowchart is represented.

\subsection{Protocol definition}

The EE-TDMA-PSO-UFC functions are divided into rounds close to LEACH (Low-energy adaptive clustering hierarchy) and BMA (Bit-Map-Assisted) protocols. Each stage involves the cluster set-up and the static state process. The EE-TDMA-PSO-UFC interface structure is shown in Figure 1. Clusters are created in the set-up phase, and the transmission of data is done in the steady-state phase to the cluster head. The static step of EE-TDMA-PSO-UFC is divided into $n$ frames, unlike with the "LEACH" and "BMA" protocols. Since not every source node has data to send, every frame is not set for a period. Transmission dates and a schedule period are contained in each frame. In each schedule period, a "TDMA" schedule is set up by the cluster head to communicate a collision-free intra-cluster. The cluster head allocates a similar time slot toward the cluster members for each round's first schedule. And for the next period of plan, different time slots are assigned to the cluster head's source nodes. Section 3.2 will discuss the duration of the time slot.

The number of source nodes in a cluster is equivalent to the division of time slots of each period's data transmission. Over the message of the assigned time slot, each source node's information and the next period's expected traffic load was sent to the cluster head. During all the time, it kept the radio off. All non-source nodes have the off conditions for radio in the period of data transmission. Source node data, like leave message, can also be given to the cluster head. Thus, depending on the number of sensors and traffic loads of sensors, the "TDMA" frame's length can be dynamically adjusted. When a structure is completed, it starts with a schedule period, and it gets repeated. From all the source nodes, the information is collected by the cluster and is sent combined and compressed data to the base station. The process starts in the next round after a predetermined period and will repeat a similar approach.

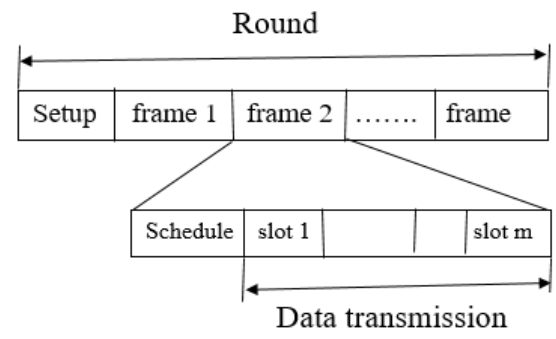

Figure 1. Illustration of an EE-TDMA-PSO-UFC frame construction

\subsection{Size of time slot}

The goal of the EE-TDMA-PSO-UFC protocol is to provide a more powerful in-cluster slot machine for wireless sensor networks. The time slot adjustment can be made based on the traffic load of cluster members and the network topology. In a frame, the time slot's source node is as follows:

$$
t i=\min \left\{\frac{l i}{C C i}, \frac{\mathrm{li}}{\sum_{j=1}^{m} \mathrm{lj}}\left(\operatorname{Tn}_{\mathrm{frame}}-\operatorname{Tn}_{\text {schedule }}\right)\right\}
$$

Here li is represented the neat frame expected traffic load of source node, $\mathrm{m}$ signifies amount of source nodes into a cluster, $\mathrm{CC}_{\mathrm{i}}$ be the capacity of channel, $\mathrm{T}_{\mathrm{i}}$ is the maximum frame duration and $T_{i \text { schedule }}$ is the schedule for the time in the frame. The time slot is assigned for source nodes with the cluster head during the schedule process and it is described in Eq. (1).

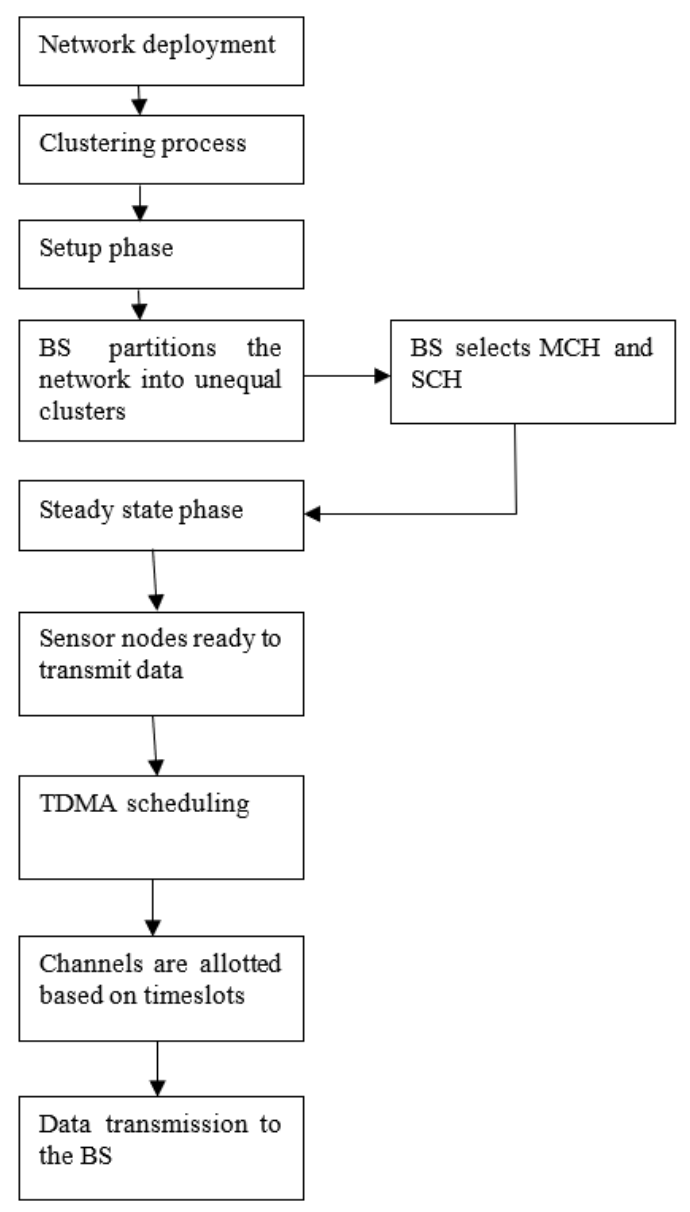

Figure 2. Flow chart 


\section{RESULTS DISCUSSION}

The "EE-TDMA-PSO-UFC" scheme is implemented using the NS-2 network simulator have proposed in this paper. We suppose random dispersal of 21 sensor nodes over a region of $1000 \times 1000 \mathrm{~m}^{2}$. Due to the connection between sensor nodes, area size, and simulation time, the author opted for 21 sensor nodes and $1000 \times 1000 \mathrm{~m}^{2}$ with a simulation time of $1200 \mathrm{~ms}$. We can also consider any number of sensor nodes and area size depending on the data level process. In this paper, there is no difference in detection, and in its abilities, static sensors are the same. The base station is located at the coordinates of ( 250 $\mathrm{m}, 250 \mathrm{~m})$. The base station received data from "MCH" and "SCH."

Table 1 displays the device parameters used in our simulations and the simulation time required for $1200 \mathrm{~ms}$. Here, the parameters are a transmission rate of 512 bytes $/ 0.5$ sec, 21 number of nodes with the area of $1000 \times 1000 \mathrm{~m}^{2}$, and radio range is $250 \mathrm{~m}$. However, AODV routing protocol and routing techniques of EE-TDMA-PSO-UFC, E-BEENISH, CEEC are implemented to verify a sensor network's efficiency.

Table 1. System parameters

\begin{tabular}{cc}
\hline Parameter & Value \\
\hline Application Traffic & CBR \\
Transmission rate & 512 bytes $/ 0.5 \mathrm{sec}$ \\
Radio range & $250 \mathrm{~m}$ \\
Simulation time & $1200 \mathrm{~ms}$ \\
Number of nodes & 21 \\
Area & $1000 \times 1000$ \\
Routing Methods & EE-TDMA-PSO-UFC, \\
Routing protocol & E-BEENISH, CEEC \\
\hline
\end{tabular}

\section{A. Performance Evaluation}

In terms of end to end delay, throughput, and the network's total energy conservation, the efficiency "EE-TDMA-PSOUFC" protocol is evaluated. If the first node (FND) or the number of nodes die, the network lifetime and energy consumption are limited. For most sparsely used WSNs, the FND metric is used. However, the death of a single node in densely distributed WSNs does not affect networking and sensing. However, the efficiency of densely-used WSN deteriorates dramatically when one-half of the number of nodes dies in CEEC and E-BEENISH.

B. Experimental Results

The network configuration is shown in Figure 3. In this case, all nodes within the range are mounted without overlap within the network.

Figure 4 describes the broadcasting process in a WSN. Based on this testing procedure, it is ensured that all nodes are transmitted within the range only after accomplishing the deployment of each node.

The structure of cluster formation is illustrated in Figure 5. Here, some nodes are forming a cluster. Accordingly, $\mathrm{SCH}$ and $\mathrm{MCH}$ have been chosen.

The transition of information among cluster heads concerning the BS is demonstrated in Figure 6. The data is primarily sent toward the cluster head by the cluster members to validate the transmitted data to the base station.

Figure 7 is shown the cluster file, which indicates the creation of various clusters along with their locations in the network, the cluster head, and data transfer links in the system.
Figure 8 displays the hop table. It describes how often the information is consumed a solitary hop or several hops to reach the destination when data transmits toward an alternate node from one node in a system. All hops in a network show in this table while data is being sent.

Figure 9 shows the transmission table, which includes the information is being sent to the network. Additionally, it informs which node has transmitted a packet to a system at a specific time.

The trace file is displayed in Figure 10 that includes a list of all variables, characteristics, data transmission processes, and energy levels.

Figure 11 illustrates the results of the end-to-end delay in a network. The proposed method of EE-TDMA-PSO-UFC has reduced the end-to-end delay than the existing methods like CEEC and E-BEENISH.

The energy utilization of a network is demonstrated in Figure 12. Based on the analysed simulation results, the reduced consumption of energy is achieved with the proposed system of EE-TDMA-PSO-UFC when compared to the EBEENISH and CEEC.

The throughput execution describes in Figure 13. It has been proved that the proposed system EE-TDMA-PSO-UFC provides enhanced throughput of a network rather than other methods such as CEEC and E-BEENISH.

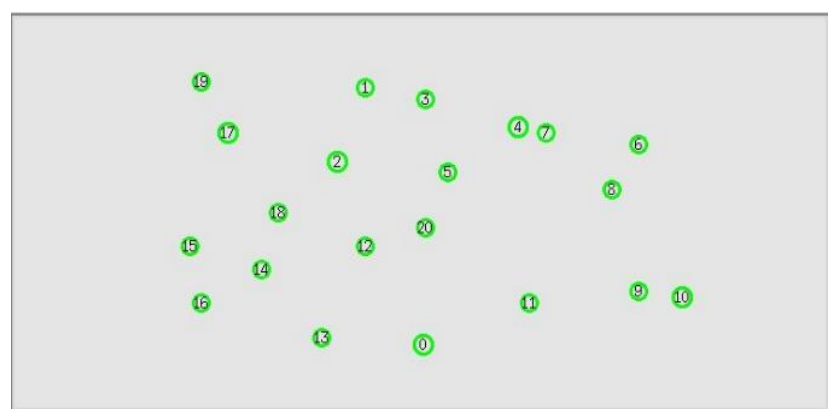

Figure 3. Network deployment

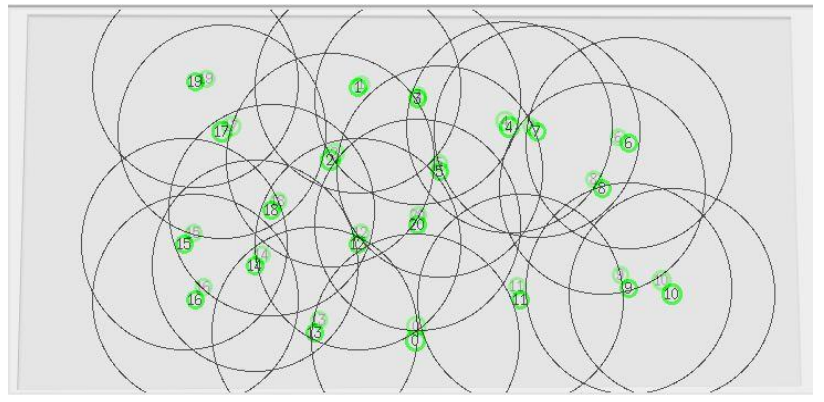

Figure 4. Three heat broadcasting process in network

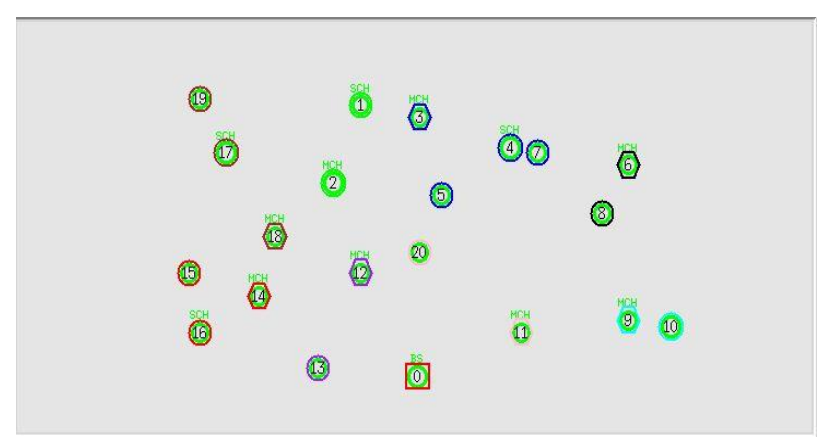

Figure 5. Cluster formation and selection of $\mathrm{MCH}$ and $\mathrm{SCh}$ 


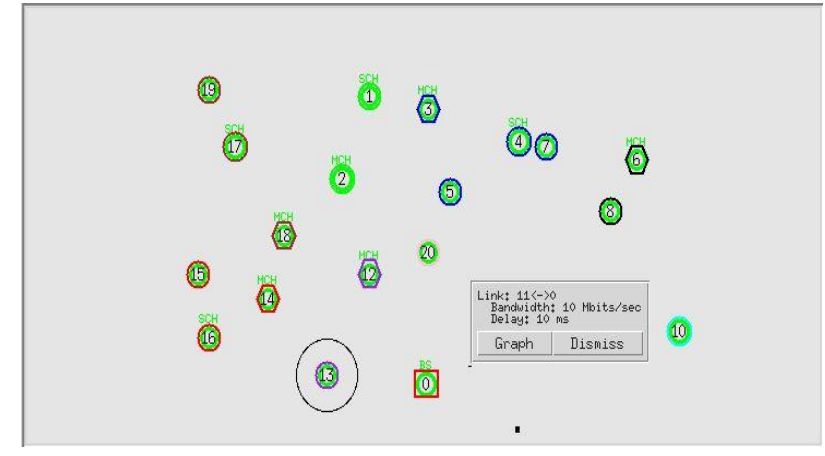

Figure 6. Three data process between cluster heads and BS

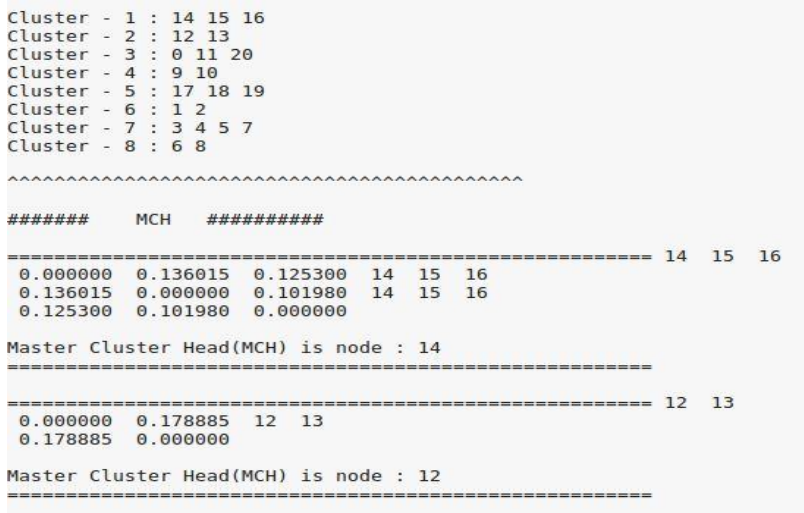

Figure 7. Cluster file

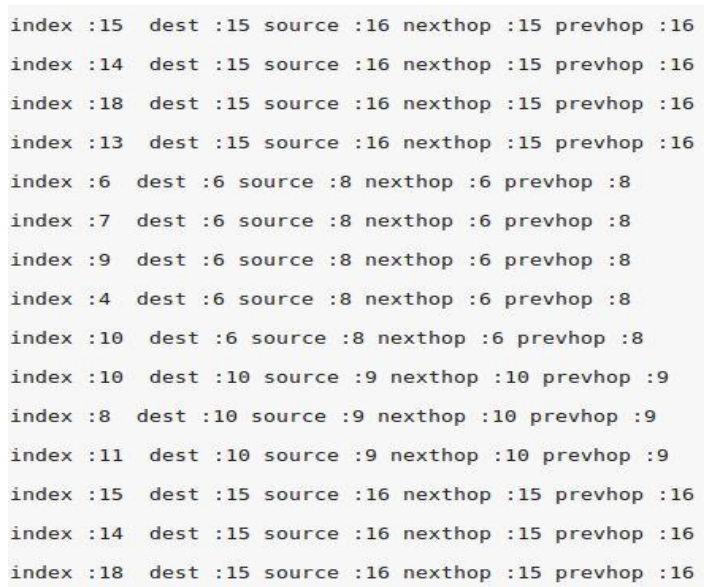

Figure 8. HOP table

\begin{abstract}
Node 2 forwards the packet to 1 at 2.520409 Node 2 forwards the packet to 1 at 2.520409 Node 12 forwards the packet to 13 at 2.592410 Node 12 forwards the packet to 13 at 2.592410 Node 16 forwards the packet to 15 at 2.736409 Node 16 forwards the packet to 15 at 2.736409 Node 16 forwards the packet to 15 at 2.852400 Node 2 forwards the packet to 1 at 2.899165 Node 9 forwards the packet to 10 at 2.970409 Node 9 forwards the packet to 10 at 2.970409 Node 9 forwards the packet to 10 at 2.977404 Node 12 forwards the packet to 13 at 2.993065 Node 8 forwards the packet to 6 at 3.078409 Node 8 forwards the packet to 6 at 3.078409 Node 8 forwards the packet to 6 at 3.134773
\end{abstract}

Figure 9. Transmission table

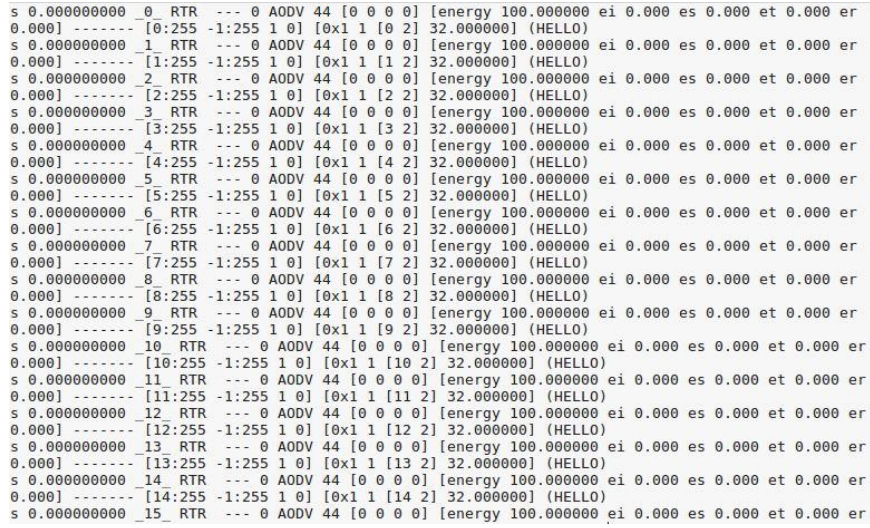

Figure 10. Trace file

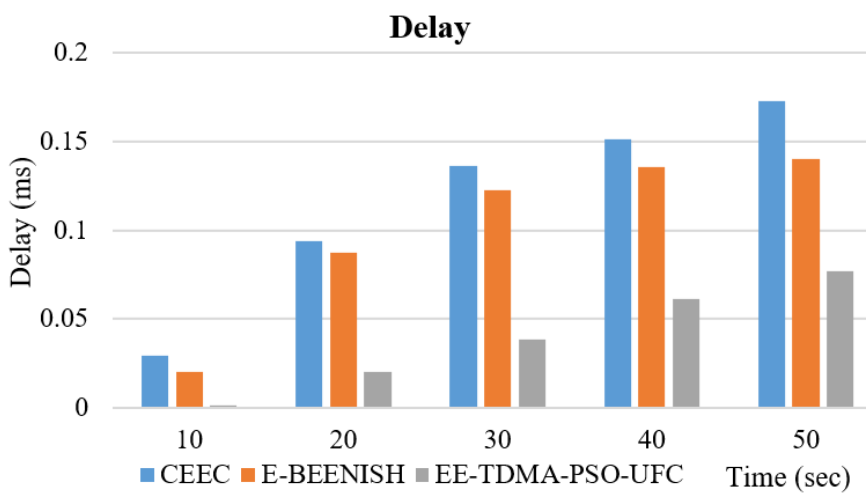

Figure 11. End-to-end delay

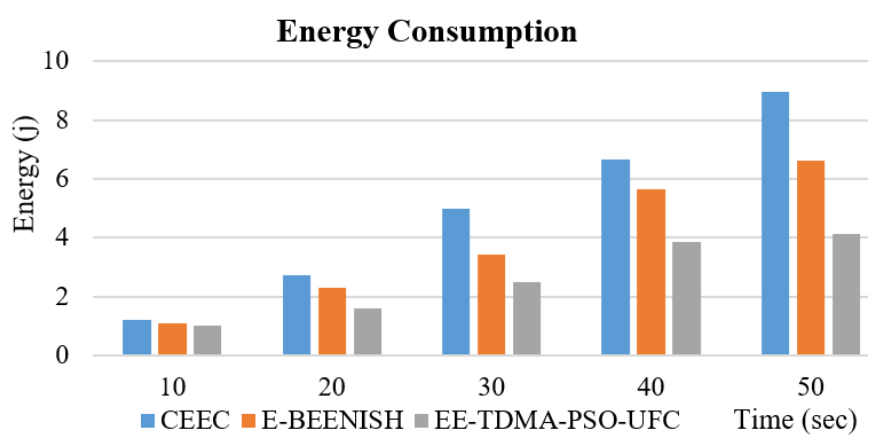

Figure 12. Energy consumption

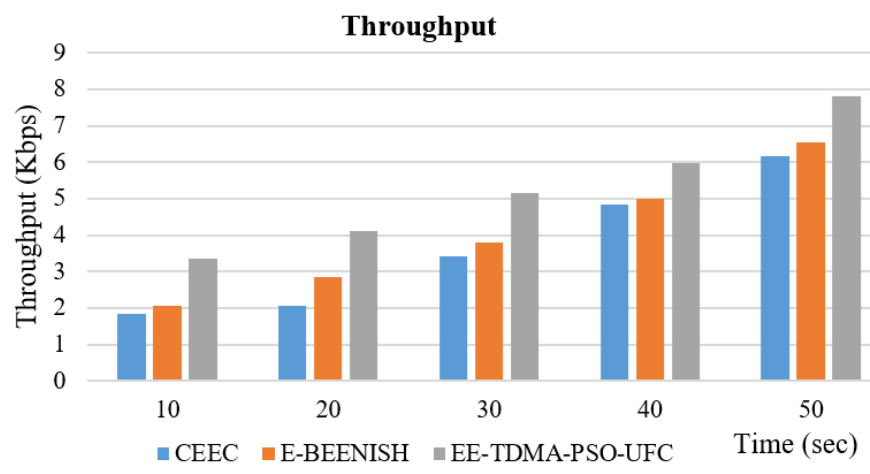

Figure 13. Throughput

\section{CONCLUSION}

This paper focuses on resolving the issue in hot point, imbalanced clustering, fault-tolerant, and timeslot problems 
with the energy-efficient scheduling of a Particle Swarm Optimizing clustering protocol for wireless sensor networks, called EE-TDMA-PSO-UFC. For determining solutions for more MCHs areas, the EE-TDMA-PSO-UFC approach aims to pick more MCHs near the base station. TDMA allocates energy-saving time slots for data transmission. The TDMA system length based on current traffic and network topology is dynamically modified by our proposed approach and reduces timeslots for energy savings. The results of the simulation are contrasted with the associated methods E-BEENISH and CEEC. The EE-TDMA-PSO-UFC process has shown that comparisons of end-to-end delay, throughput, and power consumption.

\section{REFERENCES}

[1] Cai, S., Lau, V.K. (2017). Modulation-free M2M communications for mission-critical applications. IEEE Transactions on Signal and Information Processing over Networks, 4(2):

248-263.

https://doi.org/10.1109/TSIPN.2017.2686002

[2] Paul, A.K., Sato, T. (2017). Localization in wireless sensor networks: A survey on algorithms, measurement techniques, applications and challenges. Journal of Sensor and Actuator Networks, 6(4): 24. https://doi.org/10.3390/jsan6040024

[3] Wu, D.P., He, J., Wang, H.G., Wang, C.G., Wang, R. (2015). A hierarchical packet forwarding mechanism for energy harvesting wireless sensor networks. IEEE Communications Magazine, 53(8): 92-98. https://doi.org/10.1109/MCOM.2015.7180514

[4] Zhou, M.B.Z., Thiele, L. (2018). A survey on sensor calibration in air pollution monitoring deployments. IEEE Internet of Things Journal, 5(6): 4857-4870. https://doi.org/10.1109/JIOT.2018.2853660

[5] Alshinina, R., Elleithy, K. (2018). A highly accurate deep learning based approach for developing wireless sensor network middleware. IEEE Access, 99: 1-1. https://doi.org/10.1109/ACCESS.2018.2844255

[6] ŞİMŞEK, M., Toklu, S. (2018). Application Specific Sleep-Awake Strategy for Increasing Network Lifetime in Wireless Sensor Networks. Düzce Üniversitesi Bilim ve Teknoloji Dergisi, 6(4): 1038-1046.

[7] Abidi, B., Jilbab, A., Haziti, M.E. (2017). Wireless sensor networks in biomedical: Wireless body area networks. In Europe and MENA Cooperation Advances in Information and Communication Technologies, 321329. Springer, Cham. https://doi.org/10.1007/978-3-31946568-5_33

[8] Yousef, H., Msallam, M. (2020). Variable sampling interval for energy-efficient heterogeneous precision agriculture using Wireless Sensor Networks. Journal of King Saud University-Computer and Information Sciences, $\quad 32(1)$ : $\quad 88-98 . \quad$ https://doi.org 10.1016/j.jksuci.2018.04.010

[9] Yang, O., Heinzelman, W. (2012). Modeling and performance analysis for duty-cycled MAC protocols with applications to S-MAC and X-MAC. IEEE Transactions on Mobile Computing, 11(6): 905-921. https://doi.org/10.1109/TMC.2011.121

[10] Lucchi, M., Giorgetti, A., Win, M.Z., Chiani, M. (2007). Using a UAV to collect data from low-power wireless sensors. In Proc. XIX Congresso Nazionale AIDAA, 86:
141-150.

[11] Pantazis, N.A., Nikolidakis, S.A., Vergados, D.D. (2012). Energy-efficient routing protocols in wireless sensor networks: A survey. IEEE Communications Surveys \& Tutorials, $15(2)$ : 551-591. https://doi.org/10.1145/1579114.1579148

[12] Zhang, P., Xiao, G., Tan, H.P. (2013). Clustering algorithms for maximizing the lifetime of wireless sensor networks with energy-harvesting sensors. Computer Networks, 57(14): 2689-2704. https://doi.org/10.1016/j.comnet.2013.06.003

[13] Bahbahani, M.S., Baidas, M.W., Alsusa, E. (2015). A distributed political coalition formation framework for multi-relay selection in cooperative wireless networks. IEEE Transactions on Wireless Communications, 14(12) 6869-6882. https://doi.org/10.1109/TWC.2015.2461619

[14] Engel, A., Koch, A. (2016). Heterogeneous wireless sensor nodes that target the Internet of Things. IEEE Micro, 36(6): 8-15. https://doi.org/10.1109/MM.2016.100

[15] El Alami, H., Najid, A. (2015). SEFP: A new routing approach using fuzzy logic for clustered heterogeneous wireless sensor networks. International Journal on Smart Sensing \& Intelligent Systems, 8(4). https://doi.org/10.21307/ijssis-2017-854

[16] Lee, J.S., Kao, T.Y. (2016). An improved three-layer low-energy adaptive clustering hierarchy for wireless sensor networks. IEEE Internet of Things Journal, 3(6): 951-958. https://doi.org/10.1109/JIOT.2016.2530682

[17] El Alami, H., Najid, A. (2017). (SET) Smart Energy Management and Throughput Maximization: A New Routing Protocol for WSNs. In Security Management in Mobile Cloud Computing, IGI Global, 1-28. https://doi.org/10.4018/978-1-5225-0602-7.ch001

[18] Smaragdakis, G., Matta, I., Bestavros, A. (2004). SEP: A stable election protocol for clustered heterogeneous wireless sensor networks. Boston University Computer Science Department.

[19] Qing, L., Zhu, Q., Wang, M. (2006). Design of a distributed energy-efficient clustering algorithm for heterogeneous wireless sensor networks. Computer Communications, 29(12): 2230-2237. https://doi.org/https://doi.org/10.1016/j.comcom.2006.0 2.017

[20] Saini, P., Sharma, A.K. (2010). E-DEEC-enhanced distributed energy efficient clustering scheme for heterogeneous WSN. In 2010 First International Conference on Parallel, Distributed and Grid Computing (PDGC 2010), pp. 205-210. https://doi.org/10.1109/PDGC.2010.5679898

[21] Aslam, M., Shah, T., Javaid, N., Rahim, A., Rahman, Z., Khan, Z.A. (2012). CEEC: Centralized energy efficient clustering a new routing protocol for WSNs. In 2012 9th Annual IEEE Communications Society Conference on Sensor, Mesh and Ad Hoc Communications and Networks (SECON) IEEE, pp. 103-105. https://doi.org/10.1109/SECON.2012.6275763

[22] Qureshi, T.N., Javaid, N., Khan, A.H., Iqbal, A., Akhtar, E., Ishfaq, M. (2013). BEENISH: Balanced energy efficient network integrated super heterogeneous protocol for wireless sensor networks. Procedia Computer $\quad$ Science, 19 : 920-925. https://doi.org/https://doi.org/10.1016/j.procs.2013.06.1 26 
[23] Verma, A., Mondal, R., Gupta, P., Kumar, A. (2018). Neural based energy-efficient stable clustering for multilevel heterogeneous WSNs. 2018 First International Conference on Secure Cyber Computing and Communication (ICSCCC), Jalandhar, India, pp. 208212. https://doi.org/10.1109/ICSCCC.2018.8703353

[24] Harichandan, P., Jaiswal, A., Kumar, S. (2013). Multiple aggregator multiple chain routing protocol for heterogeneous wireless sensor networks. In 2013 International Conference on Signal Processing and Communication (ICSC) IEEE, pp. 127-131. https://doi.org/10.1109/ICSPCom.2013.6719769

[25] Mahmoud, M.M., Lin, X., Shen, X. (2013). Secure and reliable routing protocols for heterogeneous multihop wireless networks. IEEE Transactions on Parallel and Distributed Systems, 26(4): 1140-1153. https://doi.org/10.1109/TPDS.2013.138

[26] Al Masri, A., Delisle, G.Y., Hakem, N. (2016). Hierarchical routing protocol for multi-level heterogeneous sensor network. In 2016 16th
Mediterranean Microwave Symposium (MMS) IEEE, pp. 1-4. https://doi.org/10.1109/MMS.2016.7803853

[27] Srilakshmi, U. (2017). Proficient interference exposure expedients to secure MANET from occurrences. IJAT, 8: 4. https://doi.org/10.4172/0976-4860.1000195

[28] Srilakshmi, U., Rao, B.S. (2018). Mitigate the routing overhead in WSN using new random walk detectors based approach. Journal of Advanced Research Dynamic Control System (International Journal), 10(4).

[29] Srilakshmi, U., Rao, B.S. (2019). A cross layer protocol to improve energy efficiency and QoS in MANET. Journal of Mechanics Continua and Mathematical Sciences, (ESCI Indexed journal, Web of Science), 14(1).

[30] SriLakshmi, U., Rao, B.S. (2019). An overhead aware multipath routing protocol for improving relay node selection in MANET. IJRTE (International Journal), 8(1).

[31] Srilakshmi, U., Yakobu, D., Jose Moses, G., Anusha, P., Laxmi Lydia, E. (2020). Energy efficient with load balancing bee ad-hoc routing protocol for manet. Test Engineering \& Management, 82. 